\title{
Raman spectroscopy of fingernails: A novel tool for evaluation of bone quality?
}

\author{
N.M. Cummins ${ }^{\text {a,* }}$, J.C.C. Day ${ }^{\text {b }}$, A. Wren ${ }^{\text {a }}$, P. Carroll ${ }^{\text {c }}$, N. Murphy ${ }^{\text {c }}$, P.M. Jakeman ${ }^{d}$ and \\ M.R. Towler ${ }^{\mathrm{a}, \mathrm{e}}$ \\ ${ }^{a}$ Clinical Materials Unit, Materials and Surface Science Institute, University of Limerick, Limerick, \\ Ireland \\ ${ }^{\mathrm{b}}$ Interface Analysis Centre, University of Bristol, Bristol, UK \\ ${ }^{\mathrm{c}}$ Department of Health, Sport and Exercise Science, Waterford Institute of Technology, Waterford, \\ Ireland \\ ${ }^{\mathrm{d}}$ Department of Physical Education and Sports Science, University of Limerick, Limerick, Ireland \\ e Inamori School of Engineering, Alfred University, Alfred, NY, USA
}

\begin{abstract}
Raman data from human fingernails has been analysed to determine if a relationship exists between spectral features and bone quality. Previous work demonstrated a relationship between the manually determined intensity of the disulphide peak (Bone Quality Test; BQT) and osteoporotic fracture. A computer program is now described that automates the BQT determination for large sample-sets. In this study the ability of the automated BQT to discriminate between fractures and controls was compared to that of bone mineral density (BMD) and biomarkers of bone remodelling. Females aged 18-67 years participated in the study $(n=159)$. Fingernails were analysed using Raman spectroscopy. Lumbar and Femoral BMD was measured by dual energy X-ray absorptiometry (DXA). Fasting venepuncture samples were analysed for osteocalcin and CTx (collagen C-telopeptides) by electrochemiluminescence. No correlation was found between the BQT and BMD or the biomarkers. The BQT and BMD were found to be equally accurate in identifying subjects with a history of fracture $(p<0.01)$ and both outperformed osteocalcin and CTx. It appears that keratin and collagen are related structural proteins that require disulphide bonding for stability. Therefore, these preliminary results suggest that Raman spectroscopy of keratin may have potential as a diagnostic tool for screening bone quality in large populations.
\end{abstract}

Keywords: Raman spectroscopy, osteoporosis, fracture, fingernails, bone density, biomarker

\section{Introduction}

Raman spectroscopy is a promising tool for evaluation of bone quality and fracture risk $[11,18,19]$. The spectroscopic parameters related to bone quality are mineral content, mineral crystallinity and collagen cross-link ratio [21]. Raman analysis of iliac crest biopsies has revealed bone tissue compositional differences in women with and without osteoporotic fracture. Fracture cases were reported to have a higher carbonate/amide I area ratio than controls in femoral trabecular samples and a higher carbonate/phosphate ratio in cortical bone samples [19], which was in agreement with previous results from infrared spectroscopy studies [6].

\footnotetext{
*Corresponding author: Dr. Niamh M. Cummins, Clinical Materials Unit, Materials and Surface Science Institute, University of Limerick, National Technological Park, Limerick, Ireland. Tel.: +353 61 234170; Fax: +353 61 338172; E-mail: niamh.cummins@ul.ie.
} 
Raman spectroscopy of nail samples has previously proved successful in archaeological [12] and forensic investigations $[1,30]$. The composition of finger and toe nail samples are influenced by several physiological and pathological processes [27]. Fingernails have recently been identified as a biomarker for esophageal cancer using infrared spectroscopy [28].

Anecdotally, osteoporosis patients have reported increased fingernail strength following pharmaceutical treatment [16]. Preliminary work by the authors has evaluated the use of Raman spectroscopy to investigate the link between fingernail protein structure (keratin) and bone protein structure (collagen) [20, $24,26]$. Both proteins require cysteine incorporation and sulphation for structural integrity. The biosynthesis of procollagen requires both intra- and inter-chain disulphide bond formation [7]. Noncollagenous proteins in the organic matrix such as osteonectin also require disulphide bonding for stability [5]. It was recently reported that postmenopausal women with osteoporosis have elevated total homocysteine (Hcy) and significantly reduced plasma cysteine (Cys) levels in comparison to their healthy counterparts [4]. The inverse relationship between total Hcy and Cys might imply that a trans-sulphuration defect could be impairing the irreversible conversion of total Hcy to Cys. The authors suggest that a low Cys concentration, possibly due to reduced flux from Hcy, may lead to less availability for collagen formation resulting in poor bone quality [4].

Changes in the organic matrix, or in the collagen molecule alone, have been observed in osteoporosis [3]. Bone strength therefore reflects the integration of two main features, bone density and bone quality [22]. Bone quality refers, in part, to the organic matrix of bone but also describes a set of characteristics that influence strength such as architecture, remodelling and damage accumulation [22]. Measurement of bone density by dual energy X-ray absorptiometry (DXA) is the current gold standard for the diagnosis of osteoporosis. However, DXA is not adequate for population screening due to cost constraints and availability. More importantly, DXA is limited in its ability to detect individuals who will fracture [23] as subjects with normal bone density can suffer fragility fractures due to factors independent of BMD, for example bone remodelling rate [14]. Biochemical markers of bone remodelling can be used as a measure of bone quality and have been reported to predict bone loss [8] and future fracture risk [14]. These markers have the advantage of being non-invasive and also respond more quickly to intervention than densitometry [29].

Significantly lower disulphide content of fingernails have been observed in subjects with a history of low-impact fracture, using the Raman spectroscopy based Bone Quality Test (BQT) [20,26]. Therefore, the aim of this study is to further investigate the validity of Raman spectroscopy as a tool in the assessment of bone health and to compare its efficacy with the other techniques currently available in clinical practice.

\section{Experimental}

\subsection{Study design and patient population}

With ethical approval and informed consent, 159 eligible females (18-67 years) participated in this cross-sectional study. Subjects were recruited by posters, email and word of mouth at the university campus and from the surrounding area. Prior to enrolment, subjects completed a questionnaire detailing medical and fracture history. A low-impact fracture was defined as a fracture resulting from a fall of standing height or less. Post-menopausal status was defined by duration of greater than 18 months post-menopause. Subjects were excluded from the study on the basis of medical conditions (other than primary osteoporosis) or treatment (bone active medication) that may affect bone or fingernail health. 


\subsection{Collection of nail samples}

Two nail clippings were taken from each subject from the free edge of the nail plate and stored in sealed microtubes. Samples were swabbed with acetone (Reagecon, Shannon, Ireland) to remove any traces of nail varnish prior to analysis.

\subsection{Raman spectroscopy}

Raman analysis (Inspector Raman Instrument, Delta $\mathrm{Nu}$, WY, USA) was performed on the underside of the fingernail. Excitation was by diode laser operating at $785 \mathrm{~nm}$. Spectra were recorded (400$1800 \mathrm{~cm}^{-1}$; Fig. 1) by securing the nail to the analysis port and performing ten scans, each with a laser exposure time of $5 \mathrm{~s}$, to improve the signal-to-noise ratio. The average of ten scans was taken as one determination. Two determinations were performed upon each of the two nails. In previous work the intensity of S-S bonding in the sample (Bone Quality Test; BQT) was evaluated manually $[20,26]$. However, manual determination of peak intensity may be influenced by the subjective assessment of the peak base, especially for spectra with varying background shapes. To preclude this, and to facilitate efficient analysis of larger data sets, a computer program was written to automate the methodology (Dr. John Day, Interface Analysis Centre, University of Bristol, UK). The program, written in Borland Delphi 7, allowed the import of a large number of spectra obtained from patients divided into control and fracture groups. The program initially normalized spectra to obtain a constant total intensity and averaged spectra for each patient before implementing a method for the determination of S-S peak width.

The background in Raman spectra is dominated by fluorescence and it was evident that this was highly variable between different samples. The peak width measurement is affected by parameters other than signal intensity. Raman peak width is dependent on variations in the length of the active bond, which may be due to strain or structural broadening caused by variations in the local crystal order [9]. A method of peak width determination based on the derivative spectra was implemented. In this the first derivative

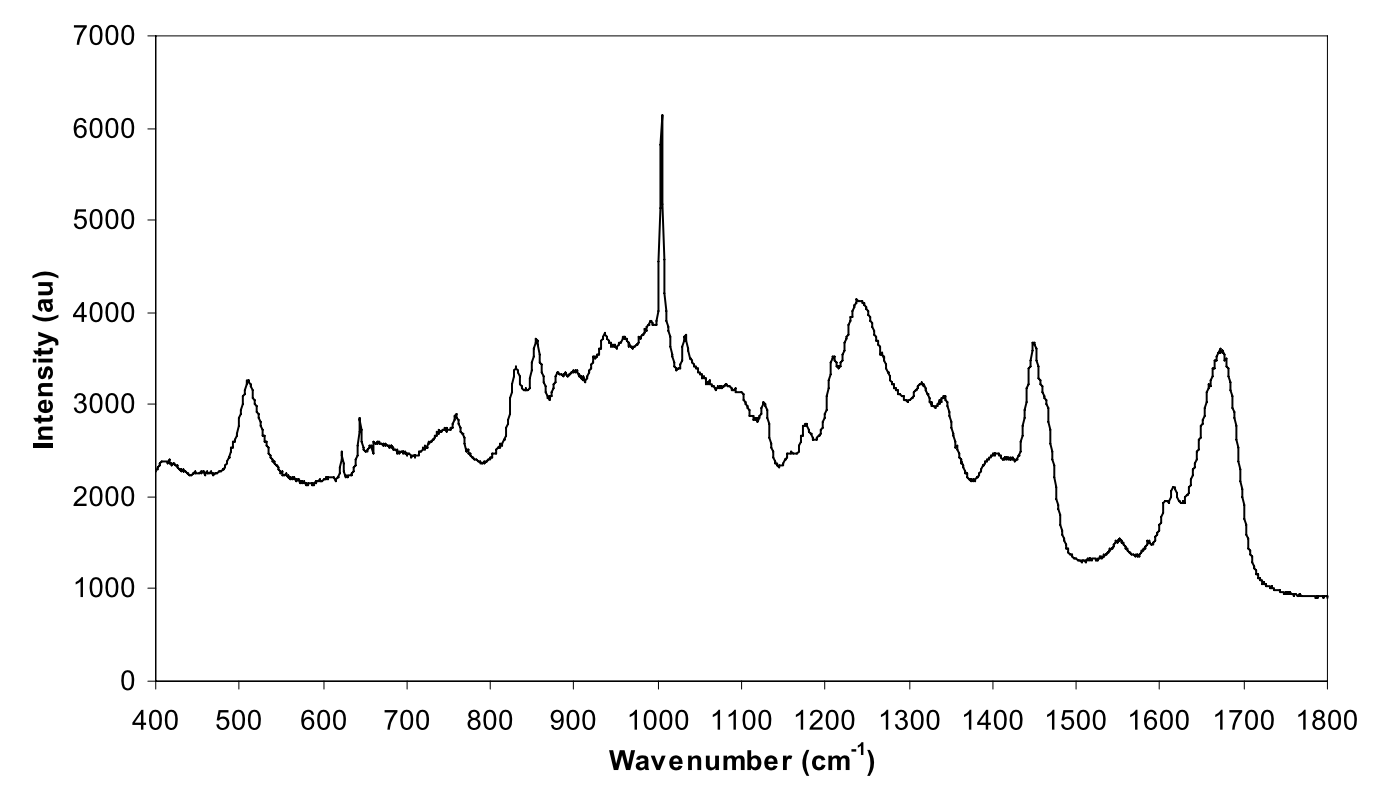

Fig. 1. Raman spectrum of a human fingernail $400-1800 \mathrm{~cm}^{-1}$. 
of the spectrum was calculated. The maximum and minimum derivative values were then found on either side of the S-S peak at $510 \mathrm{~cm}^{-1}$. These correspond to the points of steepest slope about the peak centre. The distance between these two points was used as a measure of peak width. The derivative calculation was made either as a simple arithmetic difference between consecutive points or by applying a SavitskyGolay smoothing filter to the data. Therefore, in this study the BQT was calculated as the width of the $\mathrm{S}-\mathrm{S}$ peak at $510 \mathrm{~cm}^{-1}$ for each sample.

\subsection{Bone densitometry}

Lumbar and Femoral BMD was measured by DXA (Norland Medical Systems, NY, USA) with strict adherence to recommended quality assurance practice.

\subsection{Biochemical markers of bone remodelling}

Single venepuncture samples were collected from fasting subjects and serum was analysed for bone formation (N-MID ${ }^{\circledR}$ Osteocalcin) and resorption ( $\beta$-CTx crosslaps) markers by electrochemiluminescence (Elecsys 1010, Roche, Basel, Switzerland). All values were within the reference ranges reported by the manufacturer and the coefficient of variation was $4 \%$ for osteocalcin and $5 \%$ for CTx.

\subsection{Calcium intake}

A food frequency questionnaire was used to assess calcium (Ca) intake in the population. This method has previously been reported to correlate well $(r=0.79)$ with the gold standard weighed food record [2].

\subsection{Data analysis}

Statistical analysis was performed using SPSS software (Microsoft, CA, USA). Variables were tested for normality using the Kolmogorov-Smirnov test. Depending on the normality of the distribution, Pearsons or Spearmans correlation coefficients were calculated to examine the relationship between the clinical tools. Comparisons between parameters in the fracture and control groups were performed by the Student's $t$-test or the Mann-Whitney test (MWU) as appropriate.

\section{Results and discussion}

The study population comprised 159 women of whom 81 were pre-menopausal and 78 were postmenopausal. A total of 34 fracture cases were recorded with 16 occurring in the pre-menopausal women and 18 occurring in the post-menopausal women. Menopausal status did not significantly influence BQT $(p=0.062)$, therefore further analysis was performed on the total group of 159 subjects in order to improve the power of the study. Fractures were recorded in subjects who had normal BMD, osteopenia and osteoporosis, with wrist, ankle and arm fractures being most common. The BQT was slightly higher in the high-impact fractures $(n=26)$ than in the low-impact fractures $(n=8)$ although this did not reach significance ( 37.37 vs. 35.57 , respectively, $p=0.749$ ).

No significant difference in age $(p=0.829)$, calcium intake ( $p=0.504)$ or BMI $(p=0.855)$ was observed between the control and fracture groups (Table 1). There was a higher prevalence of smokers in the fracture group $(n=8,24 \%)$ than in the control group $(n=17,14 \%)$. Smokers had significantly 
Table 1

Study group characteristics

\begin{tabular}{lccc}
\hline & Total group $(n=159)$ & Control group $(n=125)$ & Fracture group $(n=34)$ \\
\hline Mean age (SD) (years) & $45(15)$ & $46(15)$ & $44(17)$ \\
Mean Ca (SD) (mg/d) & $814(368)$ & $807(369)$ & $840(370)$ \\
Mean BMI (SD) (kg/m $\left.{ }^{2}\right)$ & $25.19(3.88)$ & $25.31(4.10)$ & $24.72(2.91)$ \\
Current smokers (number) $\left._{\text {Family history ost. (number }}^{*}\right)$ & $n=25$ & $n=17$ & $n=8$ \\
\hline
\end{tabular}

Note: ${ }^{*}$ Number of participating subjects with a family history of osteoporosis.

Table 2

Student's $t$-test and Mann-Whitney results for bone health measures in control and fracture groups

\begin{tabular}{lccc}
\hline & Control group $n=125$ & Fracture group $n=34$ & Significance \\
\hline Mean Lumbar BMD (SD) $\left(\mathrm{g} / \mathrm{cm}^{2}\right)$ & $1.032(0.165)$ & $0.947(0.172)$ & $p=0.009$ \\
Mean Femoral BMD (SD) $\left(\mathrm{g} / \mathrm{cm}^{2}\right)$ & $0.877(0.133)$ & $0.829(0.141)$ & $p=0.071$ \\
Mean BQT (SD) (a.u.) & $45.02(13.75)$ & $36.94(13.56)$ & $p=0.003$ \\
Median* Osteocalcin (SD) $(\mathrm{ng} / \mathrm{ml})$ & $27.22(11.12)$ & $27.62(11.25)$ & $p=0.735$ \\
Median* CTx (SD) (ng/ml) & $0.423(0.216)$ & $0.433(0.228)$ & $p=0.617$ \\
\hline
\end{tabular}

Note: * Data non-normally distributed therefore median value from MWU presented.

lower Lumbar BMD than non-smokers (0.938 vs. 1.028, $p=0.015)$ and Femoral BMD approached significance $(p=0.058)$. Similar findings were recently reported by Demirbag et al. in 2006 [10]. Smoking did not appear to influence disulphide levels in fingernails $(p=0.340)$. The relationship between cysteine, homocysteine and smoking currently remains unclear in the literature and requires further investigation $[17,25]$. There was no significant difference between smokers and non-smokers in serum levels of osteocalcin and CTx.

Significantly lower disulphide content of fingernails was again observed in subjects with a history of fracture and this is in agreement with previous studies $[20,24]$. In comparison to the other methods used in this study the BQT discriminated most accurately between the control and fracture cases $(p=0.003$; Table 2).

Disulphide bonding is essential for procollagen folding and stability of mature collagen [7]. These preliminary results suggest that nail keratin may be a suitable analogue for bone collagen. In this study, the BQT performed comparably with DXA (Lumbar BMD, $p=0.009$ ) which is the current gold standard for diagnosis of osteoporosis. This is in agreement with a previous study which suggests that BQT and BMD are equivalent predictors of fracture status (BQT odds ratio 0.188 vs. BMD $0.282, p<0.0005$ ) [26]. DXA evaluates the mineral phase of bone. There does not appear to be any relationship between DXA and the BQT (Lumbar BMD, $R=-0.121, p=0.130$; Fig. 2), despite both techniques being comparable in their ability to identify subjects with a history of fracture. This is likely due to the composite nature of bone because fracture risk depends on the health of both the organic and mineral phases which are evaluated separately by the two different techniques.

Biochemical markers of bone remodelling can be used to evaluate the organic phase of bone $[8,14]$. However, in this study, biomarkers did not prove to be useful in distinguishing between the fracture and control groups (Osteocalcin, $p=0.735, \mathrm{CTx}, p=0.617$ ). This could be due to the fact that single samples were analysed in this study and these biomarkers express a high level of biological variability. Seasonal and diurnal rhythms exist and biomarkers are known to be influenced by exercise, bed-rest 

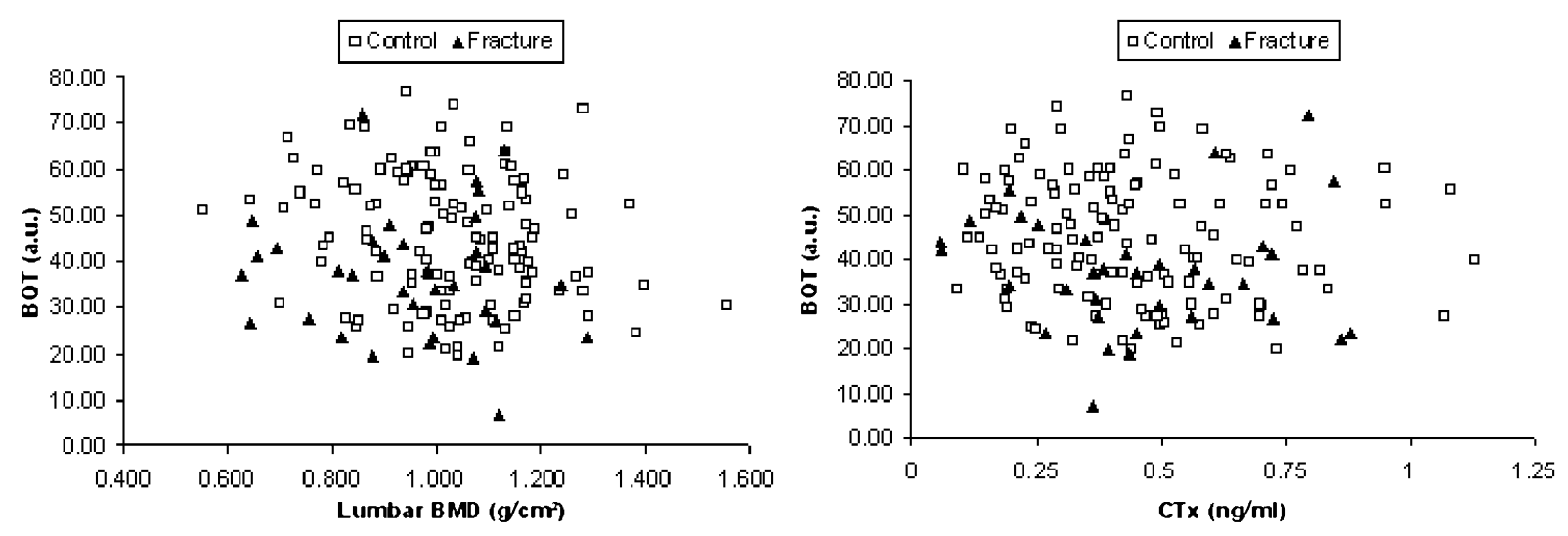

Fig. 2. Correlation of the BQT with Lumbar BMD and CTx $(n=159)$.

and extremes of diet [29]. There was no correlation between the biomarkers and the BQT (e.g., CTx, $R=-0.079, p=0.325$; Fig. 2 ) which could be attributed to the previously discussed single sample variability. It is possible that there may not be a relationship between osteocalcin and CTx and the disulphide levels of keratin, because of the bone characteristics that the BQT is assessing, but a relationship may exist with other biomarkers such as cathepsin $\mathrm{K}$, an osteoclast-derived member of the cysteine protease family that has the ability to cleave both helical and telopeptides regions of collagen [13].

There was also no relationship between the biomarkers and DXA in this study (e.g., osteocalcin vs. Femoral BMD, $R=0.101, p=0.204)$. This agrees with the literature as biochemical bone markers are reported to contribute less than $10 \%$ to the BMD variance at any skeletal site among pre-menopausal and early post-menopausal women [15].

One of the limitations of this study was the low number of fractures, particularly low-trauma fractures, in the population. A further limitation was the retrospective, cross-sectional design and relatively small sample size of the study. Based on the results obtained here it is hoped that a large-scale prospective study will be conducted in the near future which will incorporate multivariate analysis of data. This will allow the nail-bone relationship to be explored in depth by examining additional peaks in the Raman spectrum of nail and bone such as the methylene band at $1450 \mathrm{~cm}^{-1}$ and the amide I band at $1650 \mathrm{~cm}^{-1}$. Paired specimens of nail and bone from biopsies or an animal trial, may also help to shed light on the physiological link between collagen and keratin.

The use of fingernails as a biomarker would have the advantage of greater stability over biochemical markers of bone remodelling in serum or urine and also greater responsiveness than BMD as measured by DXA, due to fingernail growth rates. The absence of ionising radiation, ease of use, portability of the equipment and its cost effectiveness could make the BQT an attractive option for managing osteoporosis in the future. With further development, it appears that the BQT may have potential for monitoring osteoporosis therapy, as a pre-screen for DXA, or in combination with DXA to assist in the early identification of individuals who are at high risk of fracture.

\section{Conclusions}

Although further investigation is necessary, it appears that Raman spectroscopy of keratin may have potential for evaluating bone quality and fracture risk. The advantages of examining nail lie in its ability 
to assess properties other than those measured by DXA, alongside its accessibility and its rapid turnover, allowing change to be monitored on a more frequent basis.

\section{Acknowledgement}

The authors gratefully acknowledge the financial support of the Enterprise Ireland Innovation Partnership Fund.

\section{References}

[1] E.M. Ali, H.G.M. Edwards, M.D. Hargreaves and I.J. Scowen, Raman spectroscopic investigation of cocaine hydrochloride on human nail in a forensic context, Anal. Bioanal. Chem. 390 (2008), 1159.

[2] R.M. Angus, P.N. Sambrook, N.A. Pocock and J.A. Eisman, A simple method for assessing calcium intake in Caucasian women, J. Am. Diet. Assoc. 89 (1989), 209-214.

[3] A.J. Bailey and L. Knott, Molecular changes in bone collagen in osteoporosis and osteoarthritis in the elderly, Exp. Gerontol. 34 (1999), 337-351.

[4] M. Baines, M.B. Kredan, A. Davison, G. Higgins, C. West, W.D. Fraser and L.R. Ranganath, The association between cysteine, bone turnover, and low bone mass, Calcif. Tissue Int. 81 (2007), 450-454.

[5] M.E. Bolander, M.F. Young, L.W. Fisher, Y. Yamada and J.D. Termine, Osteonectin cDNA sequence reveals potential binding regions for calcium and hydroxyapatite and shows homologies with both a basement membrane protein (SPARC) and a serine proteinase inhibitor (ovomucoid), Proc. Natl. Acad. Sci. USA 85 (1988), 2919.

[6] A.L. Boskey and R. Mendelsohn, Infrared spectroscopic characterisation of mineralised tissues, Vib. Spectrosc. 38 (2005), 107-114.

[7] N.J. Bulleid, Novel approach to study the initial events in the folding and assembly of procollagen, Cell Dev. Biol. 7 (1996), 667-672.

[8] C.H. Chesnut, N.H. Bell, G.S. Clark, B.L. Drinkwater, S.C. English, C.C. Johnson, M. Notelovitz, C. Rosen, D.F. Cain, K.A. Flessland and N.J. Mallinak, Hormone replacement therapy in postmenopausal women: urinary N-telopeptide of type I collagen monitors therapeutic effect and predicts response of bone mineral density, Am. J. Med. 102 (1997), 29-37.

[9] N.B. Colthup, Introduction to Infrared and Raman Spectroscopy, Academic Press, Boston, MA, USA, 1990.

[10] D. Demirbag, F. Ozdemir and M. Ture, Effects of coffee consumption and smoking habit on bone mineral density, Rheum. Int. 26 (2006), 530-535.

[11] E.R. Draper, M.D. Morris, N.P. Camacho, P. Matousek, M. Towrie, A.W. Parker and A.E. Goodship, Novel assessment of bone using time-resolved transcutaneous Raman spectroscopy, J. Bone Miner. Res. 20 (2005), 1968-1972.

[12] H.G.M. Edwards, M. Gniadecka, S. Petersen, J.P.H. Hansen, D.H. Nielsen, D.H. Christensen and H.C. Wulf, NIR-FT Raman spectroscopy as a diagnostic probe for mummified skin and nails, Vib. Spectrosc. 28 (2002), 3-15.

[13] P. Garnero, O. Borel, I. Byrjalsen, M. Ferreras, F.H. Drake, M.S. McQueney, N.T. Foged, P.D. Delmas and J.M. Delaissé, The collagenolytic activity of cathepsin K is unique among mammalian proteinases, J. Biol. Chem. 273 (1998), 3234732352.

[14] P. Garnero, E. Hausherr, M.C. Chapuy, C. Marcelli, H. Grandjean, C. Muller, C. Cormier, G. Breant, P.J. Meunier and P.D. Delmas, Markers of bone resorption predict hip fracture in elderly women: the EPIDOS prospective study, J. Bone Miner. Res. 11 (1996), 1531-1538.

[15] P. Garnero, E. Sornay-Rendu, M.C. Chapuy and P.D. Delmas, Increased bone turnover in late postmenopausal women is a major determinant of osteoporosis, J. Bone Miner. Res. 11 (1996), 337-349.

[16] D. Lyons (MD), Fingernails in osteoporosis, Personal Communication, Clinical Age Assessment Unit, Mid Western Regional Hospital, Limerick, 2002.

[17] M.A. Mansoor, O. Kristensen, T. Hervig, P.A. Drabløs, J.A. Stakkestad, L. Woie, O. Hetland and A. Osland, Low concentrations of folate in serum and erythrocytes of smokers: methionine loading decreases folate concentrations in serum of smokers and nonsmokers, Clin. Chem. 43 (1997), 2192-2194.

[18] P. Matousek, E.R. Draper, A.E. Goodship, I.P. Clark, K.L. Ronayne and A.W. Parker, Noninvasive Raman spectroscopy of human tissue in vivo, Appl. Spectrosc. 60 (2006), 758-763.

[19] B.R. McCreadie, M.D. Morris, T.C. Chen, D. Sudhaker Rao, W.F. Finney, E. Widjaja and S.A. Goldstein, Bone tissue compositional differences in women with and without osteoporotic fracture, Bone 39 (2006), 1190-1195.

[20] P. Moran, M.R. Towler, S. Chowdhury, J. Saunders, M.J. German, N.S. Lawson, H.M. Pollock, I. Pillay and D. Lyons, Preliminary work on the development of a novel detection method for osteoporosis, J. Mater. Sci. 18 (2007), 969-974. 
[21] M.D. Morris and W.F. Finney, Recent developments in Raman and infrared spectroscopy and imaging of bone tissue, Spectroscopy 18 (2004), 155.

[22] National Institute of Health, Osteoporosis prevention, diagnosis and therapy, NIH Consens. Statement 17 (2000), 1-45.

[23] S.M. Ott, When bone mass fails to predict bone failure, Calcif. Tissue Int. 53 (1993), S7-S13.

[24] I. Pillay, D. Lyons, M.J. German, N.S. Lawson, H.M. Pollock, J. Saunders, S. Chowdhury, P. Moran and M.R. Towler, The use of fingernails as a means of assessing bone health: A pilot study, J. Womens Health 14 (2005), 339-344.

[25] A. Sobczak, W. Wardas, W. Zielinska-Danch and K. Pawlicki, The influence of smoking on plasma homocysteine and cysteine levels in passive and active smokers, Clin. Chem. Lab. Med. 42 (2004), 408-414.

[26] M.R. Towler, A. Wren, N. Rushe, J. Saunders, N.M. Cummins and P.M. Jakeman, Raman spectroscopy of the human nail: A potential tool for evaluating bone health?, J. Mater. Sci. 18 (2007), 759-763.

[27] P.A.H. van Noord, Selenium and Human Cancer Risk - Nail Keratin as a Tool in Metabolic Epidemiology, Thesis Publishers, Amsterdam, 1992, pp. 31-52.

[28] H.Y. Wang, Y. Lü, F. Wang, X.D. Ma, S.P. Jiang, W. Wang and C.X. Li, Study on FTIR spectra of finger nails of normal people and patients of esophagus cancer, Guang Pu Xue Yu Guang Pu Fen Xi 28 (2008), 331.

[29] N.B. Watts, Clinical utility of biochemical markers of bone remodelling, Clin. Chem. 45 (1999), 1359-1368.

[30] E. Widjaja, G.H. Lim and A. An, A novel method for human gender classification using Raman spectroscopy of fingernail clippings, Analyst 133 (2008), 493-498. 


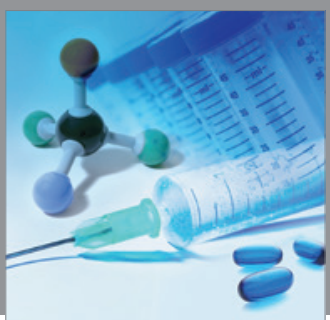

International Journal of

Medicinal Chemistry

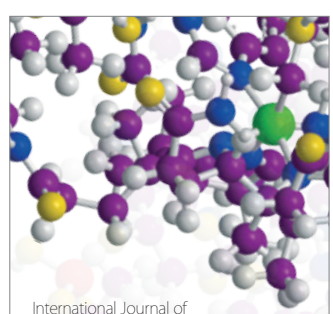

Carbohydrate Chemistry

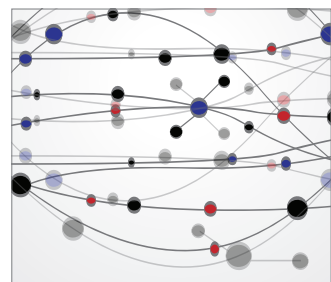

The Scientific World Journal
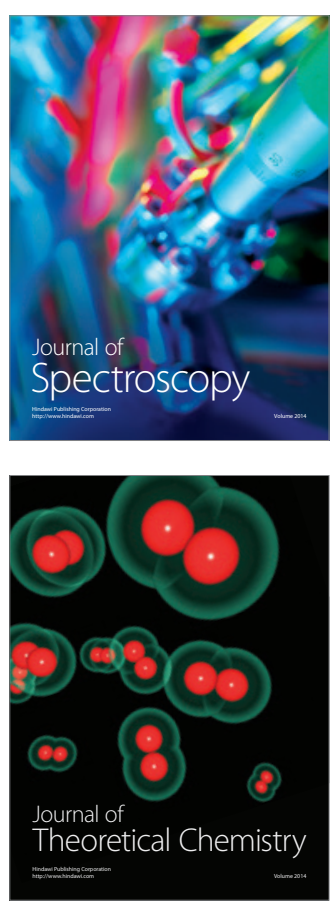
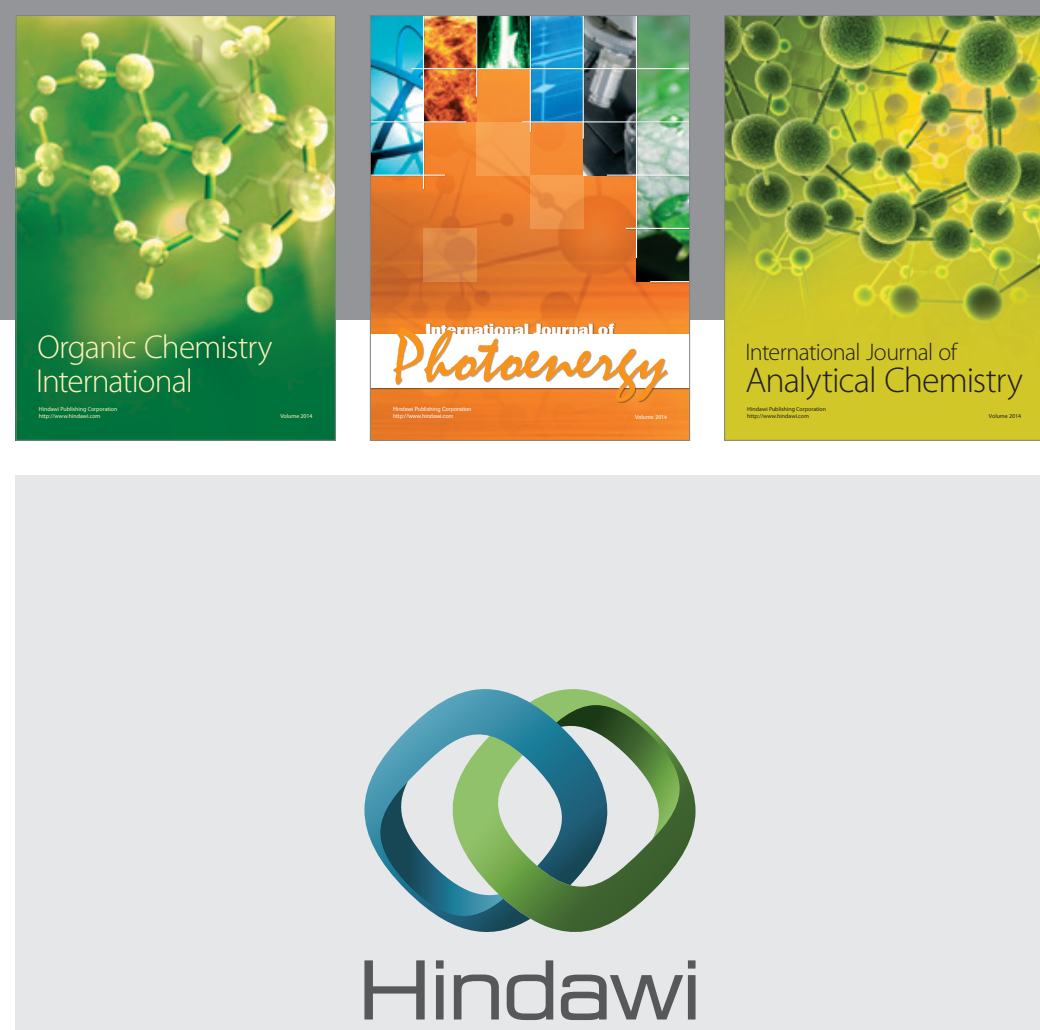

Submit your manuscripts at

http://www.hindawi.com
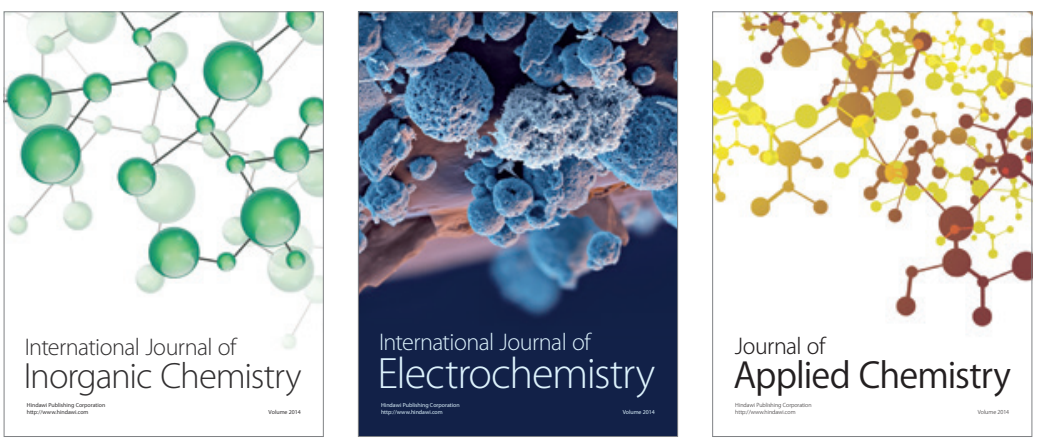

Journal of

Applied Chemistry
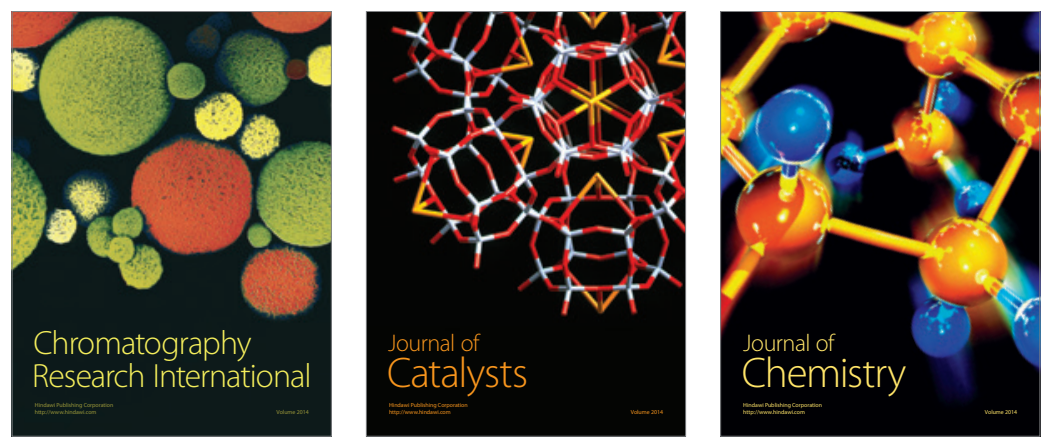
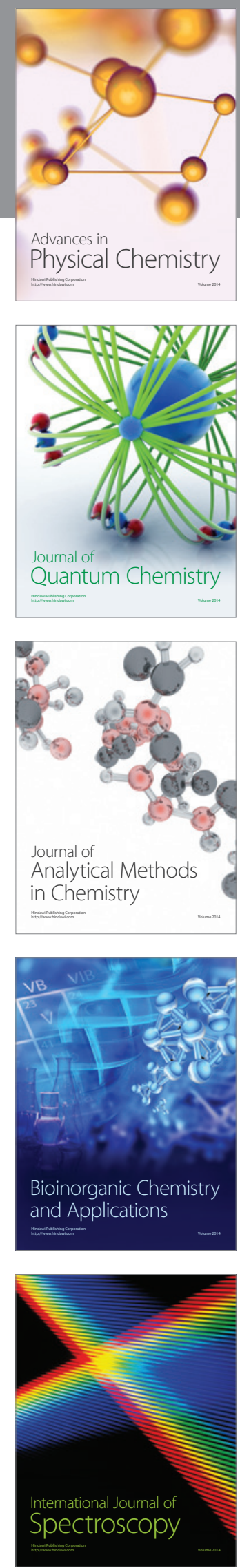